PHYSICAL REVIEW D 93, 049904(E) (2016)

\title{
Publisher's Note: Dressed scalar propagator in a non-Abelian background from the worldline formalism [Phys. Rev. D 93, 025035 (2016)]
}

Naser Ahmadiniaz, Fiorenzo Bastianelli, and Olindo Corradini

(Received 1 February 2016; published 10 February 2016)

DOI: 10.1103/PhysRevD.93.049904

This paper was published online on 28 January 2016 with an omission of two author affiliations. The author affiliations should read as

Naser Ahmadiniaz, ${ }^{1,2}$ Fiorenzo Bastianelli, ${ }^{3,4, \dagger}$ and Olindo Corradini ${ }^{1,4,5, \$}$

${ }^{1}$ Facultad de Ciencias en Física y Matemáticas, Universidad Autónoma de Chiapas, Ciudad Universitaria, Tuxtla Gutiérrez 29050, Mexico

${ }^{2}$ Center for Relativistic Laser Science, Institute for Basic Science, Gwangju 61005, Korea

${ }^{3}$ Dipartimento di Fisica ed Astronomia, Università di Bologna, Via Irnerio 46, I-40126 Bologna, Italy

${ }^{4}$ INFN, Sezione di Bologna, Via Irnerio 46, I-40126 Bologna, Italy

${ }^{5}$ Dipartimento di Scienze Fisiche, Informatiche e Matematiche, Università degli Studi di Modena e Reggio Emilia, Via Campi 213/A, I-41125 Modena, Italy

The author affiliations have been corrected as of 1 February 2016. The author affiliations are correct in the printed version of the journal. 